\title{
Testando a Eficiência Alocativa dos Municípios Paulistas ${ }^{\star}$
}

\author{
- ENLINSON MATTOS*
}

\begin{abstract}
RESUMO
Este artigo tem por objetivo testar a hipótese de eficiência alocativa para os municípios de São Paulo, usando o modelo proposto por Brueckner (1982). Neste arcabouço teórico, sob certas condições, o valor agregado das propriedades em uma comunidade que utiliza o imposto sobre propriedade é uma função com característica de U-invertido com relação ao bem público. Desta forma, comunidades eficientes, ou seja, aquelas em que o bem público é oferecido de forma ótima, apresentam níveis de gastos públicos que não afetam o valor das propriedades desta comunidade.Utilizando dados em painel e considerando variáveis instrumentais, os resultados apontam que os gastos destes municípios influenciam de forma negativa o estoque de capital (valor agregado da propriedade), sugerindo que os bens públicos estão sendo ofertados de forma ineficiente, além do nível ótimo.
\end{abstract}

\section{Palavras-Chave}

eficiência alocativa, gastos municipais, dados em painel

\begin{abstract}
This paper attempts to estimate the allocative efficiency for the cities of the state of Sao Paulo, using the model proposed by Brueckner (1982). In that model, under some conditions, the aggregate property value in a community that imposes property tax has an inverted $U$-shape function with respect to the public good. In other words, the model argues that efficient communities, i.e., those whose local government provides the public good optimally, have public expenditures not affecting the aggregate property value. Using panel data and considering instrumental variables, the results suggest that public expenses affect negatively the capital stock of the cities of the state of Sao Paulo, i.e., the public goods are overprovided in those cities.
\end{abstract}

\section{KEYWORDS}

allocative efficiency, local public spending, panel data

\author{
JEL CLASSIFICATION
}

$\mathrm{H} 42, \mathrm{H} 3 \mathrm{I}, \mathrm{H} 2 \mathrm{I}, \mathrm{H} 23$

\footnotetext{
+ Gostaria de agradecer os comentários de Marcio Holland, Fabiana Rocha, Verônica Orellano, Paulo Arvate, dois pareceristas anônimos e do Editor. Os erros remanescentes são de minha responsabilidade.

* Escola de Economia de São Paulo, Fundação Getulio Vargas. Endereço para contato: Rua Itapeva, 474, $12^{\circ}$. andar, Bela Vista, São Paulo, SP, CEP: 01332-000, Fone: 55-II-328I-3350. E-mail: enlinson@fgvsp.br.

(Recebido em novembro de 2006. Aceito para publicação em maio de 2007).
} 


\section{INTRODUÇÃO}

O debate acerca da eficiência dos gastos públicos passou a dominar a esfera de decisóes de políticas públicas nos últimos anos. Neste sentido, a questão positiva sobre se os produtos do setor público são realmente eficientes deve ser testada de forma rigorosa. Em outras palavras, é necessário determinar o nível apropriado para o tamanho do Estado, uma vez que os bens oferecidos por ele podem não estar atingindo os objetivos determinados.

Após o trabalho seminal de Samuelson (1954) que demonstra as características do equilíbrio eficiente de provisão de bens públicos, Brueckner (1979, 1980, 1982), em um contexto de bens públicos locais, demonstra, sob certas condições, que os valores agregados de propriedade em uma comunidade que utiliza imposto sobre propriedade são uma função com característica de U-invertido com relação ao bem público. O máximo ocorre no nível de produto que satisfaz a condição de Samuelson para eficiência de Pareto. Usando este resultado, Brueckner (1982), através de uma amostra cross-section de comunidades em Massachussets, mostra que estes governos locais não são grandes (over-production) nem pequenos (under-production) para prover bens públicos.

A literatura empírica geralmente busca explicar o tamanho dos governos e não testar o tamanho ótimo dos gastos em uma região ou país. ${ }^{1}$ Pode-se dividi-la em dois grupos. A primeira linha, à qual este artigo está mais relacionado, busca explicar os determinantes do tamanho dos governos. Desta forma, o objetivo destes trabalhos é medir se o nível de gastos públicos em uma região ou país corresponde a determinadas características dos agentes que residem naquela região. ${ }^{2}$ A segunda vertente pode ser entendida como uma busca para quantificar comparativamente as ineficiências das unidades que estão sobre avaliação. Assim, equipara-se um produto do bem público, como por exemplo, notas dos alunos da rede pública do Brasil ou região em algum teste, com notas de alunos da rede pública de outros países ou regiões, levando em

1 As exceções são Barro (1991) para o caso em que gastos públicos podem afetar crescimento e Brueckner (1982) no contexto de gastos municipais.

2 Com relação às estimativas sobre os determinantes dos gastos públicos no Brasil, ver, por exemplo, Mattos e Rocha (2008), que buscam identificar para o Estados brasileiros o papel da desigualdade nos gastos públicos, Botelho (2002) para os determinantes fiscais dos gastos nos Estados levando em conta o efeito flypaper, Cossio e Carvalho (2001), que estimam o efeito flypaper para os municípios brasileiros levando em conta externalidades nos gastos e Sousa e Mendes (2006a e 2006b), que apresentam estimativas sobre a demanda de serviços públicos para os municípios brasileiros. Já para a literatura internacional, a questão centra-se no tamanho dos Gastos nos Estados ou municípios sendo explicados por desigualdades sociais ou étnicas. Ou seja, o debate se dá acerca da possibilidade de unidades de federação mais desiguais (sócio/econômico/etnicamente) serem maiores (gastam mais) que outras menos desiguais. A literatura empírica não apresenta consenso. Ver por exemplo Inman (1979), Meltzer e Richard (1981) e Peltzman (1980) ou mais recentemente Husted e Kenny (1997), Milanovic (2000), Lindert (1996), Boarnet e Glazer (2002), Tanninen (1999), Bell (1989), Alesina, Baqir e Easterly (1997) e Easterly e Rebelo (1993). 
consideração o montante gasto em educação em todos as unidades de observação. Se uma região gastar muito em educação, porém seus alunos obtiverem um fraco desempenho nestes exames, pode-se concluir que existe um certo grau de ineficiência por parte do setor público neste caso. ${ }^{3}$

Alternativamente, este artigo tem como objetivo testar o modelo proposto por Brueckner (1979, 1980, 1982), utilizando dados dos municípios de São Paulo. Desta forma, busca-se avaliar se os gastos locais dos municípios do Estado de São Paulo encontram-se alocados eficientemente. Esta questão é relevante não somente do ponto de vista teórico, mas também para quantificar a eficiência de políticas públicas aplicadas aos municípios do Brasil uma vez que não existem trabalhos semelhantes. Assim, o trabalho pode ser considerado como o primeiro passo para identificar se, em média, os municípios paulistas apresentam gastos públicos eficientes, deixando aberta a questão comparativa sobre a eficiência dos gastos entre os municípios sob análise. ${ }^{4}$ Este estudo ainda se diferencia dos demais realizados em outros países, pois utiliza a metodologia de dados em painel na qual é possível controlar pelo efeito particular de cada município uma hipótese assumida no modelo, evitando estimações possivelmente viesadas ou ineficientes quando apenas dados em cross-section são utilizados. ${ }^{5}$

O trabalho está dividido em três seções além desta. A próxima seção apresenta resumidamente o modelo teórico a ser testado. A seção 3 discute os dados e apresenta a metodologia empregada; seção 4 discute os resultados da estimação; a seção seguinte apresenta os resultados referentes à extensão do modelo e a seção 6 conclui o trabalho.

\section{$2 O M O D E L O^{6}$}

Considere uma economia com consumidores com preferências idênticas e que a utilidade deles dependa do consumo de três bens: serviços de residência $(q)$, bem público (z) e um numeraire composto $(x)$. A função $u(q, z, x)$ é estritamente quasi-côncava. Assuma ainda que a utilidade é uniforme entre os municípios para os membros de

3 Ver Lovell (1993, 2000) e Kalirajan e Shand (1999). Ver, também, Sousa e Stosic (2005) e Sousa, Cribari-Neto e Stosic (2005) para os municípios brasileiros.

4 Sousa, Cribari-Neto e Stosic (2005) e Sousa e Stosic (2005) apresentam estimativas comparativas sobre a eficiência dos gastos para os municípios brasileiros.

5 Ver, por exemplo, Alesina, Baqir e Easterly (1997), que buscam estimar o efeito da diversidade étnica como determinante de gastos nos municípios dos EUA e Brueckner (1982), que busca estimar o tamanho ótimo em comunidades em Massachussets. Ambos utilizam dados em cross-section. Já para dados sobre países, Easterly e Rebelo (1993) acham uma relação positiva entre variáveis de gastos públicos e desigualdade durante o período 1970-1988 e Tanninen (1999) estabelece uma relação insignificante entre gastos e desigualdade para uma amostra com 45 países de 1970 a 1988. Estes dois últimos, apesar de possuírem a estrutura apropriada para a metodologia de dados em painel, não a utilizam.

6 Esta seção está baseada em Brueckner (1982), porém com apenas um bem público ofertado. 
cada faixa de renda, o que implica que $\hat{u}=h(y)$, sendo $y$ a renda e $h($.) alguma função com $h^{\prime}()>$.0 . O modelo adotado é o de bid-rent no qual o aluguel das casas variam, porém os indivíduos atingem o mesmo nível de satisfação independente de onde moram, dado o nível de renda. O aluguel de uma casa atrativa (alta qualidade $(q)$ ou níveis de consumo de bens públicos altos (z)) deve ser maior para garantir que os que consomem uma casa menos atrativa possuam o mesmo nível de utilidade. A restrição orçamentária dos indivíduos pode ser escrita como $x+A=y$, onde $\mathrm{A}$ denota $\mathrm{o}$ montante pago de aluguel. Pode-se, então, escrever

$$
u(z, q ; y-A)=h(y)
$$

que implicitamente determina a função de bid-rent $A=A(z, q ; y)$.

Dessa forma, os agentes maximizam a utilidade e a variável de escolha é o aluguel. Pode-se mostrar que o aluguel aumenta ao reduzir o consumo do numeraire $x$ para cancelar o aumento em utilidade de elevados níveis de $z$ ou $q$. Por último, o efeito da renda é ambíguo neste modelo. Aumentos de renda podem implicar aumentos do consumo de numeraire e conseqüentemente queda no aluguel ou vice-versa. ${ }^{7}$

Em relação ao equilíbrio nas comunidades, assuma que a arrecadação nos municípios se dê exclusivamente pelo imposto sobre a propriedade (IPTU). O valor da propriedade (preço de venda - $v$ ) é o valor descontado $(\theta)$ do aluguel subtraído dos impostos, $\tau$,

$$
v=\frac{A(z, q ; y)}{\theta+\tau}
$$

Desta forma, o valor da propriedade em uma comunidade com $n$ residências em equilíbrio,

$$
\sum_{i=1}^{n} \frac{A(z, q ; y)}{\theta+\tau}
$$

Esta equação significa que o valor da propriedade residencial agregado em equilíbrio nada mais é que o somatório de todos os aluguéis pagos nesta comunidade trazidos a valor presente, descontados os impostos.

Uma variante deste procedimento pode ser utilizada para calcular o valor da propriedade quando esta tem fins comerciais. Uma vez que aluguéis das empresas comerciais serão escalonados até eliminar os lucros, a única diferenciação é que as últimas gera-

7 O fato de a função $u($.) ser estritamente quasi-côncava garante a estrita concavidade da função $A($.) em relação a $q$ e $z$. Além disso, os argumentos da função $A($.) devem ser vistos como dependentes, o que pode ser evidenciado nos dados. Então, os agentes desta comunidade escolhem o aluguel a ser pago maximizando sua utilidade. Em equilíbrio, agentes com mesma renda possuem mesma utilidade. 
riam o valor da propriedade em uma comunidade com $m$ firmas em equilíbrio igual a $\sum_{j=1}^{m} \pi\left(s_{j}, g\right) /(\theta+\tau)$. Ou seja, dados a estrutura de insumos da firma $j\left(s_{j}\right)$ e o salário pago pelas firmas $(g)$, o aluguel desta firma se iguala ao lucro $\pi(s j, g)$. Em uma comunidade com $m$ firmas, o valor presente destes aluguéis seria então o somatório destes lucros trazidos a valor presente $(\theta)$ descontados os impostos $(\tau)$.

Suponha então que existam $m$ firmas na comunidade. O valor da propriedade total desta comunidade é o somatório do valor agregado das residências e das firmas trazidos a valor presente e descontados o imposto sobre a propriedade,

$$
P=P_{r}+P_{c}=\sum_{i=1}^{n} \frac{A(z, q ; y)}{\theta+\tau}+\sum_{j=1}^{m} \frac{\pi\left(s_{j}, g\right)}{\theta+\tau}
$$

onde $P_{r}$ denota valor da propriedade residencial, $P_{c}$ valor da propriedade para fins comerciais, $s_{j}$ denota a estrutura de insumos da firma $j$ e $g$ é o salário pago pelas firmas.

Por último, a restrição orçamentária do governo implica que o montante arrecadado com impostos deve ser suficiente para viabilizar os gastos públicos nas comunidades não cobertos pelas transferências intergovernamentais,

$$
\tau P+R=C(z, n)
$$

onde $R$ denota transferências intergovernamentais e $C($.) é a função custo assumida convexa para z e $C_{n} \geq 0$ dependendo da ocorrência ou não de congestionamento do bem público. Somando a equação (4) somente para os imóveis residenciais para $n$,

$$
P_{r}=\frac{\sum A^{i}-\tau P_{r}}{\theta}
$$

E também para as $m$ firmas,

$$
P_{c}=\frac{\sum \pi^{j}-\tau P_{c}}{\theta}
$$

Substituindo a equação (5), após somar as equações (6) e (7), a equação de interesse pode ser obtida

$$
P=\frac{1}{\theta}\left[\sum_{i=1}^{n} A\left(z, q ; y_{i}\right)+\Pi+R-C(z, n)\right]
$$


onde $\sum_{j=1}^{m} \pi_{j}\left(s_{j}, g\right)=\Pi$. Esta equação estabelece que o valor da propriedade agregado em uma comunidade homogênea é o somatório dos aluguéis residenciais e dos lucros das firmas juntamente com a contribuição do governo líquida em relação às transferências intergovernamentais $(R-C(z, n))$, trazidas a valor presente. Ou seja, o governo local pode reduzir o valor da propriedade agregado se o custo de prover bem público for muito elevado a ponto de superar os benefícios das transferências.

As estimativas apresentadas na próxima seção são comparáveis às seguintes derivadas parciais com relação às variáveis $q, y, \Pi \mathrm{e} z,{ }^{8}$

$$
\begin{aligned}
& \frac{\partial P}{\partial q_{i}}=1 / \theta A_{2}\left(z, q_{i} ; y\right)>0 \\
& \frac{\partial P}{\partial y_{i}}=1 / \theta A_{3}\left(z, q_{i} ; y\right) \stackrel{<}{=} 0 \\
& \left.\frac{\partial P}{\partial \Pi}=\frac{\partial P}{\partial R}=1 / \theta>0\right\} \\
& \frac{\partial P}{\partial z}=1 / \theta\left(\sum_{i=1}^{n} \frac{u_{z}\left(z, q_{i}, x_{i}\right)}{u_{x}\left(z, q_{i}, x_{i}\right)}-C_{1}(z, n)\right.
\end{aligned}
$$

A partir da equação (4) pode ser mostrado que as derivadas parciais de $\tau$ com relação a $q_{i}$, $\prod$ e $R$ são negativas, pois em uma comunidade com residências de alta qualidade, com grande presença de firmas e elevados níveis de transferência governamental, é possível reduzir o esforço fiscal. Desta forma, um aumento em $q_{i}$ ou $\prod$ causa um aumento direto (ver equação (4)) e indireto no valor da propriedade. Ainda, um aumento nas transferências intergovernamentais implica redução de esforço fiscal que, por sua vez, aumenta o valor agregado da comunidade. Por último, o efeito renda é indeterminado, pois este é diferente do efeito renda padrão uma vez que o consumo de casas é mantido fixo. Conseqüentemente, aumentos de renda teriam efeito ambíguo sobre os gastos com residência. ${ }^{9}$

A equação (12) estabelece que gastos públicos não afetam o valor agregado da propriedade na comunidade quando a soma das taxas marginais de substituição entre

8 Note que $A_{i}(z, q ; y)=\frac{u_{i}(z, q, y-A)}{u_{x}(z, q ; y-A)}>0, \mathrm{i}=1,2$ e $A_{3}=1-\frac{h^{\prime}(y)}{u_{3}(z, q ; y)}$ pode ser positivo ou negativo.

9 Ver Brueckner (1982), nota de rodapé 4 para a argumentação teórica deste resultado. 
o ben público (z) e o numeraire se igualar ao custo marginal (condição de ótimo de Samuelson). A estrita concavidade de $P($.) ( $A($ (.) é estritamente côncava e $C($.) é estritamente convexa) estabelece que o máximo ocorre quando as condições propostas por Samuelson valem, ou seja $\sum_{i=1}^{n} \frac{u_{z}\left(z, q_{i} ; x_{i}\right)}{u_{x}\left(z, q_{i}, x_{i}\right)}=C_{1}(z, n) \stackrel{<}{\stackrel{>}{>}} .10$

Vale ressaltar que tipo de eficiência está sendo considerado neste artigo. Brueckner (1980) mostra que com impostos distorcivos como o IPTU, o consumidor escolhe níveis de estoque de residência subótimos e a satisfação da condição de Samuelson apenas implica que a alocação de recursos é Pareto eficiente condicional ao estoque de residências subótimo. Desta forma, $\frac{\partial P}{\partial z_{k}} \neq 0$ implica que a comunidade poderia fazer melhor em equilíbrio (provendo mais ou menos bem público) condicional ao estoque de infra-estrutura.

Finalmente, é fácil ver que aumentos da população $(n)$ terão efeitos positivos ou negativos no valor agregado da propriedade dependendo quão congestionado está o bem público ofertado $\left(C_{z}>0\right)$ comparado ao incremento de aluguel. Porém, como os dados utilizados estão em termos per capita, este efeito é captado pela densidade de casas do município.

\section{IMPLEMENTAÇÃO EMPÍRICA}

\subsection{Dados}

Os dados foram extraídos do sítio do IPEA (Instituto de Pesquisa Econômica Aplicada, www.ipeadata.gov.br) e estão representados na Tabela 1 para dois períodos: 1991 e 2000. Os municípios de São Paulo foram escolhidos por dois motivos. O primeiro está relacionado a uma característica de modelo teórico onde se assume que os agentes são homogêneos por faixa de renda. Por isso, somente um Estado é utilizado. A segunda razão é que o Estado de São Paulo possui municípios com elevada arrecadação de IPTU como proporção de receitas tributárias (em média próximo de $50 \%)$ em relação ao restante do País, fato que reforça o modelo utilizado. ${ }^{11}$

10 Para o caso de se considerar que o governo municipal providencia múltiplos bens, é necessário assumir que as preferências dos agentes seja separável, ou seja $u\left(z_{1}, z_{2}, q, x\right)=\varphi\left(z_{1}, q, x\right)+\delta\left(z_{2}, q, x\right)$ o que garante a interpretação de que $\frac{\partial P}{\partial z_{k}}=0, k=1,2$ implica oferta do bem público além/aquém do nível ótimo.

11 Municípios que não existiam em 1991 são excluídos da amostra. Alternativamente, é considerado o caso em que estes assumem o valor zero para este ano. Os resultados são robustos quanto a esta questão. 
Todos os dados estão deflacionados pelo IPCA com o ano base 1991 e tratados de forma per capita. Para isso, dividem-se os dados originais pelo índice de preços calculado a partir da série do IPCA tendo como base o ano de 1991 e posteriormente pela população residente no município naquele período. De acordo com a equação (8) a ser estimada, são necessárias as seguintes variáveis: $P, Y, z, R$, П e $q$. Primeiro, a variável estoque de capital, calculada a partir da acumulação de aluguéis, é utilizada como proxy para o valor agregado da propriedade nos municípios - corresponde ao $P$ no modelo acima. Utiliza-se a renda média no terceiro quintil como a renda mediana do município $(\Upsilon)$, a despesa orçamentária e o total de receitas de transferência intergovernamentais (receita de transaçôes correntes e de capital) como gastos do governo $(z$ no modelo acima corresponde ao $G$ nas estimativas) e receitas de transferência (federal e estadual - $R$ no modelo acima e denominada Rectransf nas regressões). ${ }^{12}$ Os gastos dos municípios ainda foram divididos em despesas de habitação, saúde e educação (Habit, Sau e Educ) e demais gastos. Como proxy do lucro das firmas (П) utiliza-se a proporção de empregados total (emprego), no comércio (empcom), na indústria (empind) e serviços (empserv). ${ }^{13}$ Porém, apenas as variáveis para o comércio e serviços foram significativas para a maioria dos modelos, e como é descrito abaixo, o modelo escolhido leva em consideração apenas a variável proporção de empregados no comércio.

As variáveis utilizadas para tentar capturar a qualidade $(q)$ das residências nos municípios são: proporção de casas com água encanada e banheiro (HBath), proporção de casas com energia elétrica (enel), energia elétrica e T.V. (enelTV), energia elétrica e geladeira (enegel), proporção de casas com mais de três bens duráveis (Bensdur); por último, foi criada a variável denominada qualidade, que consiste na combinação entre as variáveis Bensdur e eneltv, altamente correlacionadas. Esta variável é utilizada como proxy da qualidade das residências nos municípios. ${ }^{14}$

Neste trabalho, os resultados referem-se à segunda alternativa, ou seja, assume-se valor zero para os municípios que não existiam em 1991.

12 Esta variável (Rectransf) busca ainda controlar, mesmo que parcialmente, a possibilidade de que a arrecadação de IPTU possa estar sujeita a uma espécie de "subestimação", uma vez que o esforço em cobrá-lo efetivamente é heterogêneo entre municípios de portes diferentes. Os municípios menores tenderiam a se esforçar menos para arrecadar tal imposto, seja por ser maior o custo político em fazê-lo ou porque as receitas tributárias tendem a ter participação menor sobre o total das receitas municipais. Agradeço esta contribuição dada por um avaliador anônimo.

13 Estes dados existem apenas para os anos de 1985 e 1995 e foram associados à base de dados de $1991 \mathrm{e}$ 2000, respectivamente.

14 Outros modelos foram testados. Uma vez que a variável qualidade está correlacionada com as outras, sua inclusão nas regressões faz com que as outras variáveis tornem-se não significativas estatisticamente. 
Finalmente, os instrumentos e controles utilizados são a proporção de casa própria (propriet), proporção da população rural em relação à urbana (proprural), proporção de pessoas com menos de 4 (anosest) e menos de 8 anos de estudo (anose) e o número de bancos $(b c o)$ nos municípios. Com relação ao aspecto geográfico dos municípios, foram consideradas a área (área), a densidade residencial que corresponde ao número de domicílios em relação à área em um município (ndomarea), uma dummy (D) caso o município pertença à região metropolitana de São Paulo, uma dummy de ano D_ano, uma dummy para as cidades que foram criadas após 1991 (D_m) e uma variável qualitativa para o tamanho das cidades (tamanho) que assume valores de 1 a 5 , dependendo do tamanho da população residente no município naquele ano, de acordo com a classificação do IBGE. ${ }^{15}$ Por último, utiliza-se a proporção de idosos com mais de 65 anos (velhos) e custo de transporte (transp) para a capital mais próxima como características demográficas. ${ }^{16}$

15 Pretende-se com isto avaliar se diferenças nos grupos de tamanho das cidades (variação discreta) afetam o valor agregado das propriedades, diferentemente da inclusão do tamanho da população (variação contínua). Alternativamente, uma dummy para cada classificação acima também foi utilizada, ou seja, uma para as cidades cuja população é inferior a 5.000 habitantes, outra dummy para as cidades cuja população está entre 5.0000 e 20.000 pessoas, outra paras cidades cuja população se encontra entre 20.000 e 100.000 e, finalmente, uma que contempla municípios com população entre 100.000 e 500.000 . Por último, como forma de dar robustez, estimamos também regressões considerando diretamente o tamanho da população ( $p o p)$ nas regressóes. Os resultados são similares e podem ser disponibilizados caso haja interesse.

16 A escolha destas variáveis segue o padrão utilizado nos artigos que buscam identificar os determinantes de Gastos nos municípios. A variável (proprural) pretende capturar a taxa de urbanização dos municípios. A variável que mede proporção de jovens nos municípios não foi estatisticamente significativa e portanto não foi incluída nas regressões. Ver, por exemplo, Mattos e Rocha (2008) para o Brasil e Case e Hines (1993) para os Estados Unidos e explicações sobre opções de coleta de dados em www.seade. gov.br. 


\section{TABELA 1 - DESCRIÇÃO DOS DADOS}

\begin{tabular}{lrr}
\hline Variável & \multicolumn{1}{c}{ Média } & Desvio padrão \\
\hline$N$ & 1290 & \\
\hline Renda & 0.0199 & 0.0223 \\
$G$ & 446.0350 & 394.0983 \\
HBath & 0.0133 & 0.0142 \\
enel & 97.7340 & 5.2637 \\
eneltv & 87.4422 & 9.9861 \\
enelgel & 87.7242 & 12.7559 \\
Bensdur & 42.1360 & 15.8533 \\
propriet & 61.7660 & 6.9182 \\
emp & 0.0001 & 0.0001 \\
$P$ & 5.5012 & 2.4044 \\
PIB & 3.8705 & 6.1964 \\
ndom & 0.2607 & 0.0735 \\
pop & 53194.8400 & 403904.1000 \\
empcom & 0.0325 & 0.0341 \\
empcomserv & 0.0363 & 0.0651 \\
empind & 0.0235 & 0.0336 \\
empserv & 0.0090 & 0.0575 \\
jovens & 0.1880 & 0.0122 \\
velhos & 0.0019 & 0.0022 \\
proprural & 0.3455 & 0.4867 \\
anosest & 41.4737 & 11.1093 \\
anose & 71.0256 & 19.4832 \\
transp & 406.0296 & 212.8579 \\
bco & 7.4140 & 62.6976 \\
desphabr & 54.2279 & 52.2212 \\
despedur & 141.4536 & 138.7638 \\
despsaur & 83.2900 & 74.9503 \\
area & 385.2600 & 352.1474 \\
ndomarea & 0.0014 & 0.0038 \\
Rectransf & 351.9892 \\
qualidade & 1665.0200 \\
\hline Fonte: &
\end{tabular}

Fonte: www.ipeadata.gov.br.

\subsection{Metodologia}

O modelo estimado é o seguinte:

$$
\begin{aligned}
& P_{i t}=\beta_{o}+\beta_{1} \text { empcom }_{i t}+\beta_{2} \text { renda }_{i t}+\beta_{3} \text { Re } \text { ctransf }_{i t}+\beta_{4} G_{i t}+\beta_{5} \text { qualidade }_{i t} \\
& + \text { crontroles }_{i t}+c_{i}+u_{i t}
\end{aligned}
$$


onde $c_{i}$ representa o efeito fixo de cada município e $u_{i t}$ o resíduo. Os controles são escolhidos se forem estatisticamente significativos.

A Tabela 2 apresenta os resultados finais da estimação. Duas alternativas são consideradas. Na primeira, assume-se que as variáveis gastos dos governos ( $G$, proxy da variável z no modelo teórico), receitas de transferências intergovernamentais (Rectransf), mediana da renda $(Y)$ e qualidade das residências $(q)$ nos municípios são exógenas. Os resultados estão representados nas colunas (1)-(3). A segunda opção leva em conta a possibilidade de estas variáveis serem endógenas e os seguintes instrumentos são utilizados: variáveis que captam o efeito do nível de emprego e lucro das firmas (empcom, empserv), a densidade residencial (ndomarea, ndom, area), proporção de idosos (velhos), proporção de residências na zona rural (proprural), o nível de educação (anosest, anose), o percentual de proprietário de casa própria (propriet) e o número de bancos (bco) do município. As estimações estão nas colunas (4)-(6). ${ }^{17}$

Como os dados estão representados em painel, utilizam-se três metodologias de estimação. A primeira faz uso do Pooled, que nada mais é que considerar todos os dados como sendo observações geradas de forma independente (colunas (1) e (4)). A segunda recorre-se ao uso de modelos de efeito fixo (colunas (2) e (5)); por último, utiliza-se o modelo de efeitos aleatórios (colunas (3) e (6)).

A seleção de modelos é feita da seguinte forma. Primeiro, compara-se os modelos com instrumentos com os demais. Neste caso, o teste de Hausman - IV sugere a rejeição de hipótese nula, ou seja, os modelos consistentes (que fazem uso das variáveis instrumentais) não devem ser rejeitados. ${ }^{18} \mathrm{O}$ próximo passo é aferir sobre a exogeneidade dos instrumentos. Para isso, implementa-se o teste de Sargan, que busca verificar se os instrumentos estão correlacionados com os resíduos da regressão. Observa-se que a hipótese nula não pode ser rejeitada, o que sugere que as variáveis utilizadas como instrumento não estão correlacionadas com os resíduos para nenhum dos casos (colunas (4)-(6)).

Isso já bastaria para concluir que as estimações referentes ao método Pooled-IV são consistentes. Porém, dois testes adicionais são realizados. O primeiro, o teste de Hausman FExRE, busca comparar o modelo de efeito fixo ( $F E-I V$ ) com o modelo de efeitos aleatórios $(R E-I V)$. O teste sugere que o modelo de efeitos aleatórios não pode ser rejeitado a $10 \%$ como nível de confiança, sugerindo que este é consistente e eficiente se comparado ao modelo de efeitos fixos. Isto tem uma implicação para o

17 Brueckner (1982) considera apenas que os Gastos Públicos, Receitas com transferências e qualidade das casas são endógenas. Considera-se aqui a possibilidade da renda $(Y)$ também ser endógena, uma vez que esta poderia estar correlacionada com o resíduo, gerando viés no estimador.

18 Apresenta-se um teste de exogeneidade das variáveis independentes ( $T$. Exogeneidade), e este teste rejeita a hipótese nula de que nã̃o há correlação entre a variável explicativa e os resíduos. Ver Wooldridge (2001, p. 285). 
modelo teórico. Neste arcabouço teórico, assume-se que os agentes são homogêneos entre as comunidades. A rejeição da hipótese de efeitos fixos pode ser entendida como um reforço ao modelo teórico uma vez que isto implica rejeição de diferenças individuais dos municípios para determinar o valor agregado da propriedade. O segundo teste busca medir a presença do efeito não-observável, teste $F-A R(1)$, ou seja, busca comparar os modelos $R E-I V$ com o Pooled-IV robusto. O teste sugere a rejeição da hipótese nula de que não há autocorrelação dos resíduos em quaisquer dos modelos estimados com variáveis instrumentais. $\mathrm{O}$ fato de o teste não rejeitar autocorrelação dos resíduos quando o efeito fixo é considerado sugere que o termo $u_{i t}$ está correlacionado. Por isso, o modelo Pooled-IV robusto à heterocedasticidade e autocorrelação não é somente consistente, mas também eficiente; é, então, o modelo escolhido para ser comentado na seção abaixo.

\section{RESULTADOS}

Conforme descrito anteriormente, o modelo escolhido é Pooled-IV (coluna (5)). De acordo com esta estimativa, os sinais dos coeficientes corroboram o modelo teórico. Por exemplo, aumentos de emprego no setor de comércio de $1 \%$ geram aumento no valor agregado da propriedade per capita de R\$ 9,65. Ainda, aumentos de $1 \%$ na proporção de casas com melhor qualidade implicam aumento no valor agregado da propriedade per capita de apenas R \$ 0,001, porém significativo estatisticamente.

A variável densidade residencial (ndomarea) também apresenta o sinal esperado, que é positivo. Esta variável captura o efeito do crescimento dos domić́lios na cidade. $\mathrm{Ou}$ seja, aumentos da proporção de domicílios por área em $1 \%$ resultam em aumento no valor agregado da propriedade per capita em $\mathrm{R} \$ 223,05$. A variável tamanho dos municípios é também significativa e positiva, porém esta variável está correlacionada com a dummy $(D)$ para regiões metropolitanas e sua interpretação tem de levar isto em consideração. ${ }^{19}$ Isto aumentaria a demanda por residências e teria como consequiência a valoração da propriedade nestes municípios.

19 Isto reforçaria a hipótese de que municípios próximos à região metropolitana têm o valor agregado da propriedade afetado positivamente, ou seja, estas cidades seriam cidades-dormitórios, onde importante parcela dos indivíduos que trabalham na capital moraria em cidades próximas. Uma explicação razoável é que isto seria o resultado do efeito spillover/borda da valoração das propriedades, porém outra metodologia teria de ser empregada. 
TABELA 2 - MODELOS ESTIMADOS

\begin{tabular}{|c|c|c|c|c|c|c|}
\hline & Pooling & $\mathrm{FE}$ & $\mathrm{RE}$ & Pooling-IV & FE-IV & RE-IV \\
\hline $\begin{array}{l}\text { Dep: Estoque de capital } \\
\text { (P) }\end{array}$ & (1) & (2) & (3) & (4) & (5) & (6) \\
\hline \multirow[t]{2}{*}{ empcom } & 6.0050 & 7.8218 & 7.1864 & 9.6574 & 10.4174 & 17.2643 \\
\hline & (2.8209) & $(1.3774$ & (1.2085) & (5.4219) & (2.9859) & $(4.0749)$ \\
\hline \multirow[t]{2}{*}{ empserv } & 1.1551 & 1.5263 & 1.3782 & & & \\
\hline & $(0.81416)$ & $(0.6022)$ & $(0.5406)$ & & & \\
\hline \multirow[t]{2}{*}{ ndomarea } & 120.0698 & 752.0926 & 134.5225 & 223.0575 & 615.2526 & 251.6406 \\
\hline & (21.1791) & (61.7382) & (12.4125) & (48.1639) & (152.6614) & (35.0909) \\
\hline \multirow[t]{2}{*}{ renda } & -26.8175 & -27.0229 & -29.2195 & -58.3432 & -64.9243 & -53.6383 \\
\hline & $(4.0010)$ & (5.5272) & (2.9225) & $(0.8848)$ & (13.2700) & (8.0162) \\
\hline \multirow[t]{2}{*}{ Rectransf } & 0.0023 & 0.0018 & 0.0024 & 0.0156 & 0.0081 & 0.0191 \\
\hline & $(0.0007)$ & $(0.0003)$ & $(0.0003)$ & $(0.0037)$ & $(0.0020)$ & $(0.0038)$ \\
\hline \multirow[t]{2}{*}{ G } & -0.00004 & -0.00004 & -0.00002 & -0.0099 & -0.0042 & -0.0145 \\
\hline & $(0.0005)$ & $(0.0003)$ & $(0.00003)$ & $(0.0041)$ & $(0.0025)$ & $(0.0043)$ \\
\hline \multirow[t]{2}{*}{ qualidade } & 0.0008 & 0.0003 & 0.0008 & 0.0013 & 0.0013 & 0.0013 \\
\hline & $(0.00004)$ & $(0.00003)$ & $(0.00003)$ & $(0.0001)$ & $(0.0003)$ & $(0.0001)$ \\
\hline \multirow[t]{2}{*}{$D$} & -0.03222 & 1.89711 & & & 1.8748 & 0.3900 \\
\hline & $(0.0819)$ & (1.2088) & & & (1.5866) & (0.1926) \\
\hline \multirow[t]{2}{*}{ D_ano } & -1.1772 & & -1.1599 & -2.813 & -2.7851 & \\
\hline & $(0.1745)$ & & $(0.1215)$ & $(0.4302)$ & (0.9961) & \\
\hline \multirow[t]{2}{*}{ Tamanho } & 0.6693 & -0.2477 & 0.6319 & 0.6232 & & 0.5276 \\
\hline & $(0.0743)$ & $(0.1531)$ & $(0.0655)$ & $(0.1269)$ & & $(0.1468)$ \\
\hline \multirow[t]{2}{*}{ C } & 0.5575 & 2.438 & 0.7505 & -0.5302 & -0.1976 & -0.5466 \\
\hline & $(0.169)$ & $(0.5325)$ & $(0.1667)$ & $(0.4307)$ & $(1.1866)$ & $(0.4840)$ \\
\hline sigma $_{u}$ & & 2.9012 & 0.7481 & & 2.2148 & 0.9263 \\
\hline sigma $_{e}$ & & 0.8461 & 0.8471 & & 1.1015 & 1.0947 \\
\hline rho & & 0.9216 & 0.4381 & & 0.8017 & 0.4172 \\
\hline$R^{2}$ & 0.7592 & 0.7981 & 0.7577 & & & \\
\hline$F(.,)$. & 265.37 & 278.82 & & 195.99 & & \\
\hline$W(. .)$. & & & 3870.2 & & 33740.29 & 1418.27 \\
\hline T. Hausman IV & & & & 85.52 & 19.02 & 68.1 \\
\hline$p$-value & & & & 0 & 0.0008 & 0 \\
\hline T. Hausman FEXRE & & & 155.29 & & & 11.79 \\
\hline$p$-value & & & 0 & & & 0.0668 \\
\hline Teste de Sargan & & & & 1.2084 & 0.1546 & 0.7108 \\
\hline$p$-value & & & & 0.9501 & 0.9986 & 0.9778 \\
\hline$F(1,1283) A R(1)$ & & & & 29.03 & 1903.19 & 13.4 \\
\hline$p$-value & & & & 0 & 0 & 0.0003 \\
\hline T. Exogeneidade F() & 30.04 & & & & & \\
\hline$p$-value & 0 & & & & & \\
\hline
\end{tabular}

Obs: Instrumentos: empcom, empser, ndomarea, ndom, area, velhos, proprural, anosest, anose, propriet, bco, emprego. Endógenas: G Rectransf renda qualidade. Erro padrão está entre parênteses. 
O efeito da variável renda sobre o valor agregado da propriedade per capita é negativo e significativo. Note que de acordo com o modelo teórico adotado (bid-rent) isto poderia ser possível, pois o efeito renda é diferente neste caso. Mantendo a qualidade das residências fixa, ou seja, o consumo deste bem é fixo, variações na renda teriam efeito de reduzir o montante gasto com o bem residência. ${ }^{20}$

Finalmente, para as variáveis de finanças públicas (Rectransf e $G$ ) ocorre o seguinte: de acordo com a teoria, aumentos nas transferências intergovernamentais (Rectransf) causam aumentos no valor agregado da propriedade per capita. Ou seja, para cada $\mathrm{R} \$ 1,00$ de aumento nas transferências per capita gera-se um aumento de R \$ 0,015 no valor agregado da propriedade per capita. E o resultado mais importante deste trabalho aponta que os governos municipais paulistas estão produzindo bens públicos acima do ponto ótimo. Aumentos de gastos per capita de R $\$ 1,00$ causam decréscimos no valor da propriedade destes municípios em média de $\mathrm{R} \$ 0,009$.

Este resultado é importante por dois aspectos. Primeiro, aponta que os municípios paulistas gastam demais e que uma redução nestes gastos teriam impacto positivo na valoração da cidade. ${ }^{21}$ Isto pode ocorrer porque municípios 'inchados' tendem a ser ineficientes e isto poderia estar sendo captado nos resultados. Segundo; este resultado propõe uma agenda de pesquisa para os municípios de São Paulo para estimar as ineficiências e impactos dos gastos municipais ou ainda verificar que tipos de gastos podem ser considerados eficientes. Esta última questão é abordada na próxima seção.

\section{EXTENSÃO DO MODELO: COMPONENTES DOS GASTOS}

Esta seção busca explicar de forma mais detalhada os diferentes efeitos dos componentes dos gastos municipais no valor agregado da propriedade nos municípios paulistas. A Tabela 3 apresenta os resultados da estimação onde o modelo estimado corresponde ao Pooled-IV; porém, consideram-se os componentes dos gastos municipais separadamente nas regressões. Neste sentido, são considerados três componentes: Gastos com habitação (Habit), educação $(E d u c)$ e saúde (Sau) e os demais gastos. ${ }^{22}$

20 Por exemplo, quando se utiliza estática comparativa mantendo a qualidade das residências constante, um aumento de renda não pode ser convertido em melhora nem aumento na residência, apenas no montante gasto na mesma. Neste caso, o efeito renda pode gerar um efeito tanto positivo quanto negativo sobre o valor da propriedade.

21 Note que a variável impostos - IPTU - está implicitamente sendo captada pelo coeficiente dos gastos do governo. Em outras palavras, o fato de o coeficiente de $G$ ser negativo pode ser interpretado como conseqüência de elevados impostos, o que, por sua vez, reduz o valor agregado das propriedades nos municípios.

22 Estes são selecionados porque se apresentam como importantes instrumentos de política pública. No entanto, outras composições foram utilizadas e os resultados podem ser disponibilizados, caso necessário. 
São considerados 6 modelos. O primeiro inclui os três componentes (Habit, Educ e San) bem como as demais despesas. No segundo, foram excluídas as despesas com saúde por serem estatisticamente não significativas. As demais despesas passam a incluir estes gastos. O terceiro modelo exclui as demais despesas, novamente por apresentarem elevado desvio padrão. Os modelos (4), (5) e (6) incluem apenas os gastos com habitação, educação e saúde, respectivamente, e as demais despesas se ajustam em cada caso com cautela. A presença de multicolinearidade pode afetar não somente o desvio padrão das estimativas, mas em alguns casos os coeficientes das variáveis. ${ }^{23}$ A Tabela 4 mostra a correlação incondicional das variáveis. Nota-se que os gastos em habitação parecem possuir um menor grau de correlação com as outras variáveis comparativamente, porém ainda elevado. Por exemplo, a correlação entre os gastos com habitação e as demais despesas (excluindo apenas os gastos em questão) é de 0,64. Este número vai para 0,89 para os gastos com educação e 0,8 para os gastos com saúde.

Antes de discutir os resultados, vale notar que os componentes dos gastos municipais do Estado de São Paulo são altamente correlacionados e as estimativas devem ser interpretadas com cautela.

Em primeiro lugar, é importante salientar que a inclusão dos componentes de gastos ao invés dos gastos agregados não altera o sinal dos coeficientes estimados das demais variáveis (exceção da constante para o modelo (5)); porém, observa-se que a constante muda a significância estatística. Ela passa a ser significativa nos modelos (4) e (6). Em relação à variável tamanho, observa-se que esta passa a ser não significativa para todas as especificações.

23 Ver Greene (2003). 
TABELA 3 - EXTENSÃO DOS MODELOS ESTIMADOS

\begin{tabular}{|c|c|c|c|c|c|c|}
\hline & $\begin{array}{l}\text { Full } \\
\text { (1) }\end{array}$ & $\begin{array}{c}\text { exc. Sau } \\
\text { (2) }\end{array}$ & $\begin{array}{c}\text { exc. Demais } \\
\text { (3) }\end{array}$ & $\begin{array}{c}\text { Habit } \\
(4)\end{array}$ & $\begin{array}{c}\text { Educ } \\
(5)\end{array}$ & $\begin{array}{l}\text { Sal } \\
(6)\end{array}$ \\
\hline empcom & $\begin{array}{r}9.5569 \\
(6.0486)\end{array}$ & $\begin{array}{r}8.3540 \\
(5.5425)\end{array}$ & $\begin{array}{r}7.2699 \\
(4.3677)\end{array}$ & $\begin{array}{l}13.8270 \\
(7.6575)\end{array}$ & $\begin{array}{r}5.9127 \\
(4.3590)\end{array}$ & $\begin{array}{r}14.6949 \\
(7.459441)\end{array}$ \\
\hline ndomarea & $\begin{array}{l}135.8730 \\
(64.1804)\end{array}$ & $\begin{array}{l}125.7940 \\
(63.7838)\end{array}$ & $\begin{array}{r}114.6620 \\
(53.7752)\end{array}$ & $\begin{array}{l}186.5454 \\
(68.6314)\end{array}$ & $\begin{array}{l}188.1761 \\
(41.1776)\end{array}$ & $\begin{array}{r}216.2323 \\
(52.11711)\end{array}$ \\
\hline renda & $\begin{array}{r}-44.2549 \\
(9.5963)\end{array}$ & $\begin{array}{r}-43.7820 \\
(9.8646)\end{array}$ & $\begin{array}{r}-45.2738 \\
(9.0687)\end{array}$ & $\begin{array}{r}-31.7216 \\
(8.1785)\end{array}$ & $\begin{array}{r}-66.9344 \\
(9.1677)\end{array}$ & $\begin{array}{r}-56.4372 \\
(12.41767)\end{array}$ \\
\hline Rectransf & $\begin{array}{r}0.0196 \\
(0.0038)\end{array}$ & $\begin{array}{r}0.0185 \\
(0.0036)\end{array}$ & $\begin{array}{r}0.0174 \\
(0.0029)\end{array}$ & $\begin{array}{r}0.0207 \\
(0.0038)\end{array}$ & $\begin{array}{r}0.0151 \\
(0.0040)\end{array}$ & $\begin{array}{r}0.0211 \\
(0.0060)\end{array}$ \\
\hline Habit & $\begin{array}{r}0.0295 \\
(0.0167)\end{array}$ & $\begin{array}{r}0.0327 \\
(0.0155)\end{array}$ & $\begin{array}{r}0.0353 \\
(0.0131)\end{array}$ & $\begin{array}{r}0.0152 \\
(0.0133)\end{array}$ & & \\
\hline Educ & $\begin{array}{l}-0.0464 \\
(0.0164)\end{array}$ & $\begin{array}{r}-0.0510 \\
(.0107)\end{array}$ & $\begin{array}{r}-0.0522 \\
(0.0091)\end{array}$ & & $\begin{array}{r}-0.0373 \\
(0.0087)\end{array}$ & \\
\hline Sau & $\begin{array}{l}-0.0157 \\
(0.0272)\end{array}$ & & & & & $\begin{array}{l}-0.0690 \\
(0.0240)\end{array}$ \\
\hline Demais & $\begin{array}{l}-0.0031 \\
(0.0070)\end{array}$ & $\begin{array}{r}-0.0023 \\
(0.0065)\end{array}$ & & $\begin{array}{l}-0.0202 \\
(0.0038)\end{array}$ & $\begin{array}{r}0.0032 \\
(0.0055)\end{array}$ & $\begin{array}{c}-0.0074 \\
(0.0067)\end{array}$ \\
\hline qualidade & $\begin{array}{r}0.0012 \\
(0.0002)\end{array}$ & $\begin{array}{r}0.0010 \\
(0.0002)\end{array}$ & $\begin{array}{r}0.0010 \\
(0.0002)\end{array}$ & $\begin{array}{r}0.0009 \\
(0.0001)\end{array}$ & $\begin{array}{r}0.0012 \\
(0.0001)\end{array}$ & $\begin{array}{r}0.0017 \\
(0.0002)\end{array}$ \\
\hline tamanho & $\begin{array}{r}0.5842 \\
(0.3055)\end{array}$ & $\begin{array}{r}0.4966 \\
(0.2281)\end{array}$ & $\begin{array}{r}0.4516 \\
(0.1949)\end{array}$ & $\begin{array}{r}0.9244 \\
(0.1422)\end{array}$ & $\begin{array}{r}0.3299 \\
(0.1656)\end{array}$ & $\begin{array}{r}0.8379 \\
(0.2650)\end{array}$ \\
\hline$D_{-} C$ & & & & $\begin{array}{r}-0.3813 \\
(0.1732)\end{array}$ & & \\
\hline$D$ & & & & $\begin{array}{r}186.545 \\
(68.6314)\end{array}$ & $\begin{array}{r}0.2802 \\
(0.1690)\end{array}$ & $\begin{array}{r}0.5954 \\
(0.2578)\end{array}$ \\
\hline$D \_a$ & & & & & $\begin{array}{l}-1.6318 \\
(0.5884)\end{array}$ & $\begin{array}{r}-1.55466 \\
(0.8260)\end{array}$ \\
\hline c & $\begin{array}{r}-0.8178 \\
(0.7672)\end{array}$ & $\begin{array}{l}-0.4951 \\
(0.5365)\end{array}$ & $\begin{array}{l}-0.3415 \\
(0.4506)\end{array}$ & $\begin{array}{r}-0.3813 \\
(0.1732)\end{array}$ & $\begin{array}{r}0.0036 \\
(0.5204)\end{array}$ & $\begin{array}{l}-1.8405 \\
(0.9159)\end{array}$ \\
\hline$F(. .)$. & 140.54 & 134.1 & 139.03 & 139.03 & 140.76 & 66.81 \\
\hline$F(1,1277)$ & 0.68 & 0.38 & 0.21 & 0.12 & 5.59 & 7.02 \\
\hline
\end{tabular}

Obs: Instrumentos: empcom, empser, ndomarea, ndom, area, velhos, proprural, anosest, anose, propriet, bco, emprego. Endógenas: G Rectransf renda qualidade. Erro padrão está entre parênteses. 


\section{TABELA 4 - CORRELAÇÃO DAS VARIAÁVEIS GASTOS MUNICIPAIS}

Demais (6) Demais (4) Demais (5) Demais (2) Demais (1) Sau Educ Habit

\begin{tabular}{|c|c|c|c|c|c|c|c|}
\hline Demais (6) & 1 & & & & & & \\
\hline Demais (4) & 0.9892 & 1 & & & & & \\
\hline Demais (5) & 0.9825 & 0.9831 & 1 & & & & \\
\hline Demais (2) & 0.9669 & 0.9825 & 0.9871 & 1 & & & \\
\hline Demais (1) & 0.9396 & 0.9372 & 0.9458 & 0.9714 & 1 & & \\
\hline Sau & 0.8078 & 0.8615 & 0.8556 & 0.8364 & 0.6822 & 1 & \\
\hline Educ & 0.9488 & 0.9508 & 0.8994 & 0.8765 & 0.8069 & 0.8358 & 1 \\
\hline Habit & 0.7065 & 0.6405 & 0.7061 & 0.5835 & 0.5008 & 0.6404 & 0.6851 \\
\hline
\end{tabular}

Os coeficientes das variáveis empcom, renda, Rectransf e qualidade permanecem relativamente próximos aos estimados na Tabela 2. Ocorre apenas uma redução do coeficiente ndomarea (sem troca de sinal) e também um ajuste na constante. Mais importante, os coeficientes das variáveis componentes dos gastos: Habit, Educ e Sau apontam para interessantes resultados. Em qualquer dos modelos escolhidos o coeficiente dos gastos em habitação (Habit) é positivo, significativo e próximo de 0,03 (no modelo 4 este valor vai para 0,015 ). Ou seja, para cada $\mathrm{R} \$ 1,00$ de aumento de gastos em habitação per capita há um aumento no valor agregado da propriedade em aproximadamente $\mathrm{R} \$ 0,03$, o que sugere que estes gastos também não se encontram alocados eficientemente. Estão aquém do nível ótimo. Já os gastos com educação apresentam coeficientes negativos, significativos e próximos de $\mathrm{R} \$ 0,05$. Uma análise similar mostra que estes gastos se encontram alocados além do ótimo. Os gastos com saúde também apresentam sinal negativo, porém somente são significativos no último modelo. Finalmente, os demais gastos somente foram estatisticamente significativos para o modelo (4) onde possui sinal negativo. Já para o modelo 6, apesar de estatisticamente insignificante, o sinal negativo deste coeficiente sugere que quando gastos em educação e habitação são considerados conjuntamente, o efeito negativo do primeiro sobre o valor agregado da propriedade per capita prevalece reforçando o resultado da seção anterior. ${ }^{24}$

\section{CONCLUSÃO}

Este artigo busca testar a hipótese de eficiência alocativa para os municípios de São Paulo, usando o modelo proposto por Brueckner (1982). Neste arcabouço teórico, sob certas condições, os valores agregados da propriedade em uma comunidade que utiliza imposto sobre propriedade são uma função com característica de U-invertido

24 Novamente, estas estimativas devem ser interpretadas com cuidado. Por exemplo, deve-se assumir que a multicolinearidade não afeta o sinal dos coeficientes. 
com relação ao bem público. Os resultados apontam que os gastos dos governos locais dos municípios paulistas são significativos e negativos para explicar a determinação do valor agregado das propriedades, o que sugere que, em média, para estes municípios, os gastos públicos são alocados acima do ponto ótimo, independente do método escolhido. Além disso, uma análise para gastos desagregados mostra que, sob certas condições, gastos em saúde e educação estão sendo alocados além, enquanto gastos com habitação estão aquém do nível eficiente. ${ }^{25}$

Uma possível interpretação para estes resultados é que estes gastos poderiam estar sendo alocados de forma ineficaz por não estarem atingindo os objetivos determinados e então aumentos nestes gastos implicariam mais ineficiência e uma valoração menor da propriedade per capita nos municípios. Uma análise mais detalhada seria necessária para investigar as possíveis causas do problema. Vale ressaltar que gastos elevados implicam impostos correspondentes e como gastos em educação representam uma parcela razoável dos gastos, esta variável pode estar captando o efeito negativo de elevado IPTU nas cidades.

Em relação ao modelo teórico, vale dizer que os dados não representam perfeitamente a hipótese assumida de que a única fonte de arrecadação é o IPTU. É sabido que os municípios arrecadam um montante razoável de ISS e outras contribuições. Isto poderia prejudicar a comparação dos resultados com o modelo teórico e também na interpretação dos resultados. ${ }^{26}$ Porém, os resultados mostrados no apêndice, onde subamostras contendo municípios cuja arrecadação do IPTU é superior a 65\% e 75\% do montante arrecadado, sugerem que isto não afeta as conclusões deste artigo.

Em suma, uma vez assumidas as hipóteses do modelo, os resultados sugerem que os municípios paulistas estão alocando gastos públicos acima do nível ótimo gerando ineficiência na alocação dos bens. Para atingir o ponto máximo da curva de U-invertido estes teriam de reduzir o tamanho dos municípios. Isto, por sua vez, sugere redução de impostos.

\section{REFERENNCIAS}

ALESINA, A.; BAQIR, R.; EASTERLY, W. Public good and ethnic divisions. Quartely Jornal of Economics, v. 114, n. 4, p. 1243- 1285, 1997.

25 Observe que este resultado é condicional à amostra dos municípios do Estado de São Paulo para este período. Novos testes teriam de ser implementados para identificar o padrão em outros Estados.

26 Por exemplo, pode-se ter um município que não cobre IPTU, apenas ISS o que inviabiliza toda análise, pois um imposto sobre serviços recai como um imposto sobre lucro. Isto não afetaria o valor da propriedade agregada diretamente. 
BARRO, R. Government spending in a simple model of endogenous growth. Journal of Political Economy, v. 98, issue 5, part 2, S103-S125, 1991.

BELL, C. R. Between anarchy and leviathan: a note on design of federal states. Journal of Public Economics, v. 39, p. 207-222, 1989.

BOARNET, M.; GLAZER, A. Federal Grants and yardstick competition. Journal of Urban Economics, v. 52, p. 53-64, 2002.

BOTELHO, R. Determinantes do comportamento fiscal dos estados brasileiros. 2002. Dissertação (Mestrado), FEA/USP.

BRUECKNER, J. Property values, local public expenditure, and economic efficiency. Journal of Public Economics, v. 11, p. 223-245, 1979.

. Property value maximization and public sector efficiency. Journal of Urban Economics, $v$. 14, p. 1-15, 1980.

. A test for allocative efficiency in the local public sector. Journal of Public Economics, v. 19, p. 311-331, 1982.

CASE, A.; HARVEY, S.; HINES JR, J. Budget spillovers and fiscal policy interdependence: evidence from the states. Journal of Public Economics, v. 52, p.285307,1993 .

COSSÍO, F.A.B.; CARVALHO, L.M. de. Os efeitos expansivos das transferências intergovernamentais e transbordamentos espaciais das despesas públicas: evidências para os municípios brasileiros - 1996. Pesquisa e Planejamento Econômico, v. 31, n. 1, 2001.

EASTERLY, W.; REBELO, S. Fiscal policy and economic growth: an empirical investigation. Journal of Monetary Economics, v. 32, p. 417-458, 1993.

GREENE, W. Econometric Analysis. 5th. Edition, Prentice Hall, 2003.

HUSTED, T. A.; KENNY, L. W. The effect of expansion of the voting franchise on the size of government. Journal of Political Economy, v. 105, p. 54-82, 1997.

INMAN, R. P. The fiscal performance of local governments: an interpretative review, In: MIEISZKOWSKI, P.; STRASZHEIN, M. (ed.). Current issues in Urban Economics. Jonhs Hopkins, 1979, p. 270-321.

KALIRAJAN, K. P.; SHAND, R. Frontier production functions and technical efficiency measures. Journal of Economic Surveys, v. 13, n. 2, p. 149-172, Apr. 1999.

LINDERT, P. H. What limits social spending? Explorations in Economic History, v. 33, p. 1-34, 1996.

LOVELL, C. Production frontiers and productive efficiency. In: FRIED, H.; LOVELL, C.; SCHMIDT, S. (ed.). The measurement of productive efficiency techniques and applications. New York: Oxford University Press, 1993.

. Measuring efficiency in the public sector. In: BLANK, Jos L. T. (ed.). Public provision and performance. Amsterdam: North-Holland, 2000. 
MATTOS, E.; ROCHA, F. Income inequality, spillover effect, and local size of the government: empirical evidence from Brazilian states. Journal of Economic Studies, forthcoming, 2008.

MELTZER, A.; RICHARD, S. A rational theory of the size of the government. Journal of Political Economy, v. 89, p. 914-927, 1981.

PELTZMAN, S. The growth of government. Journal of Law and Economics, v. 19, p. 211-240, 1980.

SAMUELSON, P. The pure theory of public expenditures. Review of Economics and Statistics 36, p.387-389, 1954.

SOUSA, M. C. S; MENDES, C. Demand for locally provided public services within the median voter's framework: The case of the Brazilian municipalities. Applied Economics, forthcoming, 2006a.

. Estimando a demanda por serviços públicos nos municípios brasileiros. Revista Brasileira de Economia, 2006b. No prelo.

SOUSA, M. C. S.; STOSIC, B. D. Technical efficiency of the Brazilian municipalities: correcting nonparametric frontier measurements for outliers. Journal of Productivity Analysis, 24, p.155-179, 2005.

SOUSA, M. C. S.; CRIBARI-NETO, F; STOSIC, B. Explaining DEA technical efficiency scores in an outlier corrected environment: The case of public services in Brazilian municipalities. Brazilian Review of Econometrics 25, p.287-313, 2005.

TANNINEN, H. Income inequality, government expenditures, and growth. Applied Economics, v. 31, p. 1109-1117, 1999.

WOOLDRIDGE, J. Econometric analysis of cross section and panel data. MIT press, 2002. 


\section{APÊNDICE 1 - AVALIANDO A EFICIÊNCIA ALOCATIVA DOS MUNICÍPIOS PAULISTAS}

\section{A.1 Municípios cuja Arrecadação com Iptu Está Acima de 65\% E 75\% do Total da Arrecadação}

A Tabela $\mathrm{Al}$ apresenta as estimativas utilizando o método Pooled-IV onde são considerados apenas os municípios com arrecadação elevada de IPTU (acima de 65\% e 75\% da receita tributária). As conclusões são qualitativamente semelhantes às descritas no artigo, porém a variável empind substitui a variável empcom como proxy do lucro das firmas.

TABELA AI - EXTENSÃO - ELEVADOS IPTU

\begin{tabular}{lrr}
\hline & IPTU>65\% & IPTU $>75 \%$ \\
& da receita & da receita \\
\hline$N$ & 131 & 81 \\
empind & 36.6043 & 143.1357 \\
& $(17.2092)$ & $(26.8093)$ \\
ndomarea & & 439.1667 \\
& & $(214.1774)$ \\
renda & -15.3838 & -2.3589 \\
& $(8.9568)$ & $(6.6709)$ \\
Rectransf & 0.0127 & 0.0074 \\
& $(0.0028)$ & $(0.0037)$ \\
G & -0.0041 & -0.0049 \\
& $(0.0019)$ & $(0.0015)$ \\
qualidade & 0.0005 & 0.0002 \\
& $(0.0002)$ & $(0.0002)$ \\
tamanho & 1.0918 & 0.4519 \\
& $(0.3345)$ & $(0.2295)$ \\
dyear & -0.1798 & 3.0464 \\
& $(0.9309)$ & $(0.6911)$ \\
C & -1.5419 & -0.7284 \\
& $(0.6387)$ & $(0.4607)$ \\
& 83.5000 & 102.5000 \\
& 0.7762 & 0.8987 \\
\hline
\end{tabular}

